\title{
Experimental Verification of Low-Frequency Noise Effects at the Onset of Oscillations in Planar Gunn Diodes
}

\author{
O. García-Pérez ${ }^{1}$, Y. Alimi ${ }^{2}$, A. Song ${ }^{2}$, I. Iñiguez-de-la-Torre ${ }^{1}$, S. Pérez ${ }^{1}$, J. Mateos $^{1}$, T. González ${ }^{1}$ \\ ${ }^{1}$ Departamento de Física Aplicada \\ Universidad de Salamanca \\ Salamanca, Spain \\ ${ }^{2}$ School of Electrical and Electronic Engineering \\ University of Manchester \\ Manchester, United Kingdom
}

\begin{abstract}
In this work, the presence of an anomalous increase of low-frequency noise associated with the initiation of the oscillatory regime in Gunn diodes has been studied and experimentally evidenced. When incipient instabilities begin to appear in the device, its intermittent nature drastically enhances the noise at frequencies well below that of the oscillation. For higher bias voltages, the oscillation becomes purer and the associated low-frequency noise disappears.
\end{abstract}

Keywords - Gunn devices; III-V semiconductor materials; lowfrequency noise; noise measurement.

\section{INTRODUCTION}

Gunn diodes are commonly used as a source of highfrequency oscillations in radiofrequency and microwave systems. Recent works have shown their feasibility to operate up to several hundreds of $\mathrm{GHz}[1]-[3]$. Moreover, some theoretical studies predict structures able to oscillate up to $\mathrm{THz}$ frequencies [4].

Such devices present a threshold voltage above which the oscillatory regime is activated. However, in the transition between the static and dynamic states there exists an intermediate bias range for which anomalous noise effects appear at very low frequencies. For higher bias voltages, when the oscillation is more stable, such noise disappears. This effect has been observed in narrow-band measurements of GaAs devices [5]-[6] and also reproduced by Monte Carlo simulations for a variety of semiconductor materials ( $\mathrm{InN}$, InGaAs, InP and GaN) [7]-[9].

The origin of such a critical enhancement of the lowfrequency noise is attributed to the coexistence of both oscillatory and non-oscillatory states occurring with a random period when the oscillation begins to be formed [10]. The aim of this work is to provide an analysis of this phenomenon, by combining results from both Monte Carlo simulations and experimental measurements performed in InGaAs Gunn diodes.

\section{Monte CARlo Simulations}

A one-dimensional InGaAs diode with an active region of $4 \mu \mathrm{m}$ length and doped $2 \times 10^{16} \mathrm{~cm}^{-3}$ has been simulated using a home-made software based on the Monte Carlo particle method. The simulation makes use of a simplified model not attempting to exactly reproduce the results of a real device. Nevertheless, these results provide valuable information to better understand the low-frequency noise mechanisms present in a Gunn structure. Moreover, the ideality of the model allows to associate the observed low-frequency noise response directly with the Gunn effect, discarding other effects due to non-idealities that may be present in an experimental setup with real samples.

The simulated current spectral densities are plotted in Fig. 1, for a frequency range between 1.5 and $150 \mathrm{GHz}$. When no bias is applied to the device, the current density is flat and corresponds to the thermal noise. For $V=1.6 \mathrm{~V}$ the noise floor grows up, and the oscillation peak begins to appear at around $50 \mathrm{GHz}$. For a voltage of $1.65 \mathrm{~V}$ the oscillation peak is clearly observable, as well as its higher frequency harmonics, while at low frequencies, a prominent noise hump appears, concentrated below $10 \mathrm{GHz}$. In a final step, increasing the voltage up to $1.7 \mathrm{~V}$ makes the oscillation peak sharper; however, the low-frequency hump vanishes at that point.

The behavior of the low-frequency current density as a function of the bias voltage is represented in Fig. 2. As it can be observed, a noticeable increase of the low-frequency noise appears around $V=1.65 \mathrm{~V}$. This point corresponds with the appearance of the first oscillations in the device. Subsequently, when the oscillation peak is well established for higher voltages, such low-frequency noise disappears.

One of the main advantages of Monte Carlo simulations is the acquisition of the instantaneous currents flowing through the device in the time domain. This information is represented in Fig. 3, and can be used in this case to qualitatively understand the device performance in the ranges previously described. Firstly, for low voltages (i.e., $V=1 \mathrm{~V}$ ), no oscillation is observed in the current, which corresponds with a constant value in the noise spectrum ("static state"). For intermediate voltages (i.e., $V=1.65 \mathrm{~V}$ ), the device initiates an unstable regime, but the oscillations are intermittent in time, as it can be observed in the second plot of Fig. 3 ("noisy oscillations"). The random period associated to such intermittences is what is observed in form of noise increase at low-frequencies. Finally, 
for high voltages (i.e., $V=2.5 \mathrm{~V}$ ) the current describes an almost purely periodic signal, without any associated lowfrequency noise component in the frequency spectrum ("pure oscillations"). Equivalent results were previously obtained by others in the literature for different materials, as in the case of [8] for GaAs and InP devices.

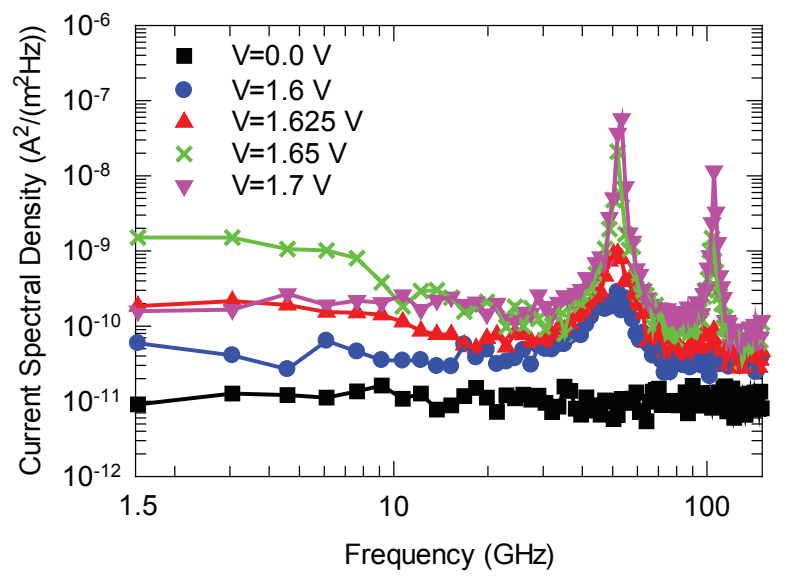

Fig. 1. Simulated current spectral density of a InGaAs Gunn device for different DC voltages.

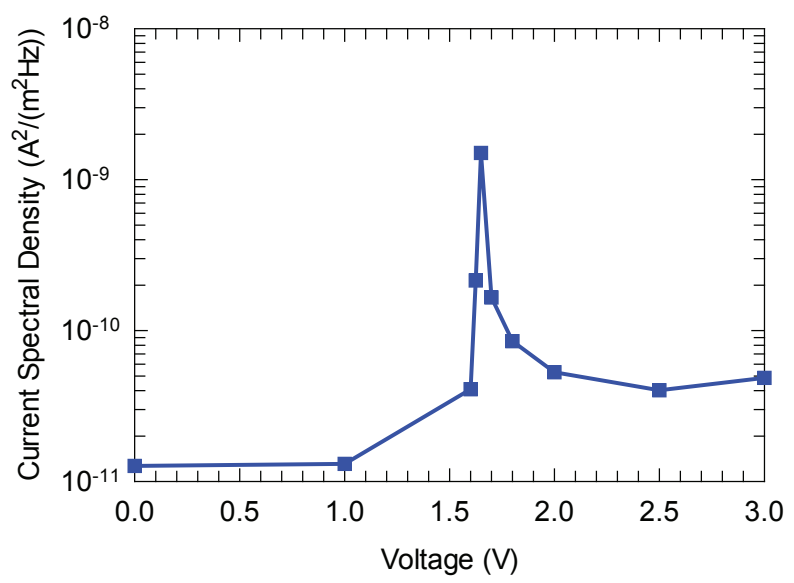

Fig. 2. Simulated noise spectral density as a function of the bias voltage in the InGaAs Gunn device at $3 \mathrm{GHz}$.

\section{EXPERIMENTAL MEASUREMENTS}

In order to study the spectral characteristics of a real Gunn device, measurements of the power spectral density of a set of InGaAs planar diodes have been carried out for the wide frequency range between $10 \mathrm{MHz}$ and $43.5 \mathrm{GHz}$. The measurements have been performed using a PNA-X N5244A with Option 029 from Agilent Technologies [11]. The setup is represented in Fig. 4. The connection to the devices is made by means of a ground-signal-ground high frequency probe. The devices are based on a structure equivalent to the one

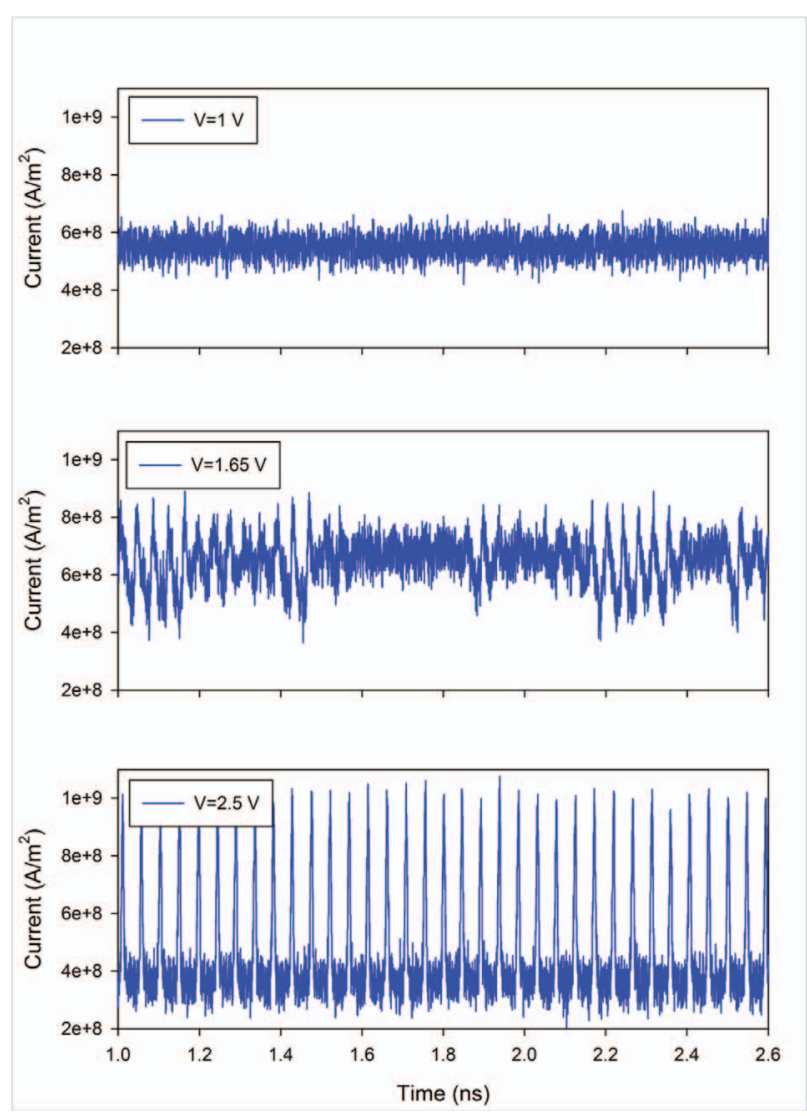

Fig. 3. Simulated current-time series of the InGaAs Gunn device for voltages $V=1 \mathrm{~V}$ ("static state"), $V=1.65 \mathrm{~V}$ ("noisy oscillations") and $V=2.5 \mathrm{~V}$ ("pure oscillations").

presented in [1], with channel lengths around $3.1 \mu \mathrm{m}$ and oscillations taking place at about $30 \mathrm{GHz}$. After a calibration step, the losses of the coaxial cable and the probe are eliminated from the measurement, so the reference plane for the characterization is at the edge of the coplanar lines that connects with the diode.

The results for a given device are shown in Fig. 5. When no voltage is applied to the structure, the power spectra is flat, with an approximate value of $-174 \mathrm{dBm} / \mathrm{Hz}$. That value corresponds with the available power density due to thermal noise of any passive device at $300 \mathrm{~K}$ [12]. For $V=5 \mathrm{~V}$, the noise floor increases and $1 / f$ noise appears at lower frequencies. For a voltage of $V=5.5 \mathrm{~V}$ an oscillation peak is visible in the upper part of the spectrum. When the bias voltage reaches $8 \mathrm{~V}$, the oscillation peak is stablished at around $30 \mathrm{GHz}$, and also a noise hump appears at around 400 $\mathrm{MHz}$, well below the frequency of oscillation. This anomalous low-frequency noise hump does not correspond to well-known white or $1 / f$ noise, since the frequency dependence is completely different. For higher bias voltages, when the oscillation peak is purer, the anomalous low-frequency noise disappears. 


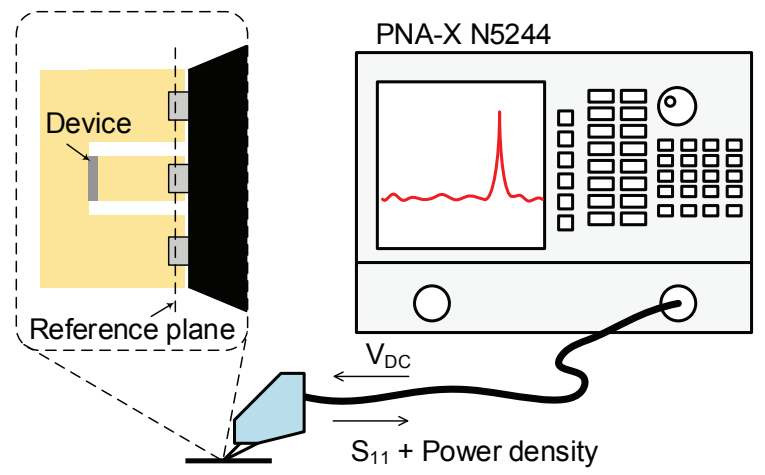

Fig. 4. Experimental setup for the measurement of the power spectral density of the planar Gunn diodes.

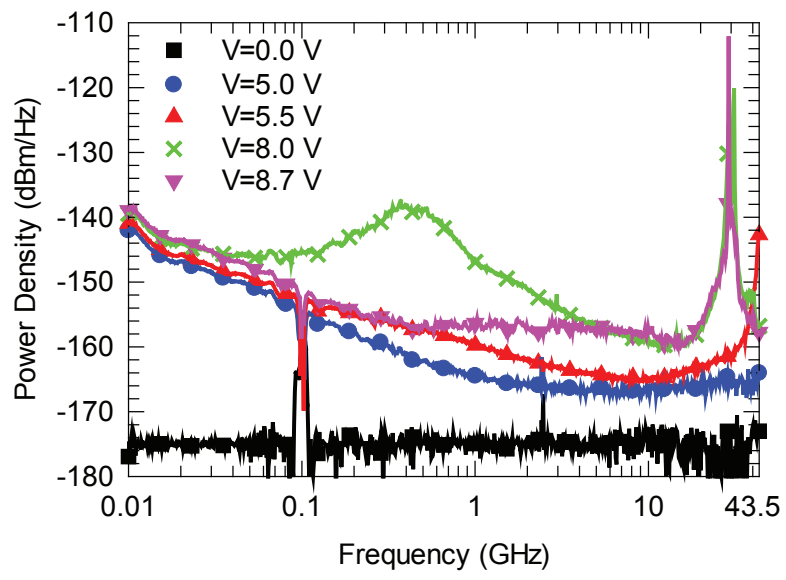

Fig. 5. Measured power spectral density of a $3.1 \mu \mathrm{m}$ length planar Gunn diode for different $\mathrm{DC}$ voltages.

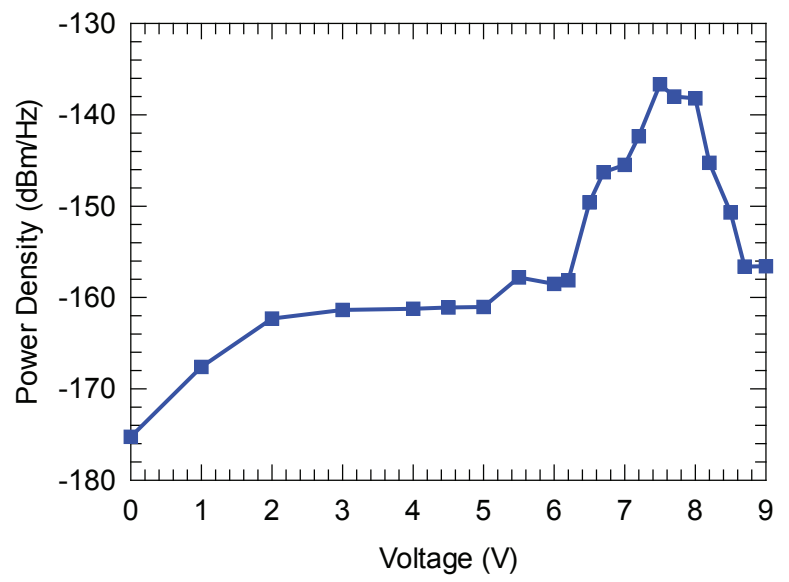

Fig. 6. Measured noise power spectral density as a function of the bias voltage of the $3.1 \mu \mathrm{m}$ length planar Gunn diode at $400 \mathrm{MHz}$.
The evolution of the low-frequency noise, i.e., at $400 \mathrm{MHz}$, with respect to the bias voltage is presented in Fig. 6. The behavior is equivalent to the one observed in the simulations in Fig. 2. For low voltages, when the device is not oscillating, the low-frequency noise presents low values. In this case, it corresponds to $1 / f$ noise, so the curve is roughly proportional to the DC current. When the device begins to present some high-frequency instabilities, the low-frequency noise grows up two orders of magnitude due to the generation of intermittent oscillations. Finally, for higher voltages, when the oscillation is a stable periodic signal, the low-frequency noise decays. An equivalent response was obtained in more samples of similar lengths, demonstrating the repeatability of the described phenomenon.

\section{CONCLUSION}

The presence of enhanced low-frequency noise associated with the initiation of oscillations in Gunn devices has been demonstrated. Both simulated and experimental results have been provided to better understand the phenomenon. In practice, this low-frequency noise effects can be used as indicator to predict very high frequency oscillations in setups where a direct detection is challenging, as in the case of (sub-)THz systems.

\section{ACKNOWLEDGMENT}

This work has been partially supported by the Spanish MINECO through project TEC2013-41640-R, and by the Consejería de la JCyL through project SA052U13. Ó. GarcíaPérez wants to thank the European Social Fund (ESF) for financing his postdoctoral contract.

\section{REFERENCES}

[1] C. Li, A. Khalid, S. H. Paluchowski-Caldwell, M. C. Holland, G. M. Dunn, I. G. Thayne and D. R. S. Cumming, "Design, fabrication and characterization of $\mathrm{In}_{0.23} \mathrm{Ga}_{0.77}$ As-channel planar Gunn diodes for millimetre wave applications," Solid-State Electron., vol. 64, pp. 67-72, 2011.

[2] A. Khalid, C. Li, V. Papageorgiou, G. M. Dunn, M. J. Steer, I. G. Thayne, M. Kuball, C. H. Oxley, M. Montes Bajo, A. Stephen, J. Glover and D. R. S. Cumming, "I $\mathrm{I}_{0.53} \mathrm{Ga}_{0.47}$ As Planar Gunn Diodes Operating at a Fundamental Frequency of $164 \mathrm{GHz}$," IEEE Electron Device Lett., vol. 34, pp. 39-41, Nov 2013.

[3] A. Khalid, G. M. Dunn, R. F. Macpherson, S. Thoms, D. Macintyre, C. Li, M. J. Steer, V. Papageorgiou, I. G. Thayne, M. Kuball, C. H. Oxley, M. Montes Bajo, A. Stephen, J. Glover and D. R. S. Cumming, "Terahertz oscillations in an $\mathrm{In}_{0.53} \mathrm{Ga}_{0.47} \mathrm{As}$ submicron planar Gunn diode," J. Appl. Phys., vol 115, p. 114502, 2014.

[4] S. Pérez, T. González, D. Pardo, J. Mateos, "Tehahertz Gunn-like oscillations in InGaAs/InAlAs planar diodes," J. Appl. Phys., vol. 103, p. 094516, 2008.

[5] K. Matsuno, "Critical fluctuation in GaAs in DC electric field," Phys. Lett. A, vol. 31, pp. 335-336, Mar. 1970.

[6] S. Kabashima, Y. Yamakazi, T. Kawakubo, "Critical fluctuation near threshold of Gunn instability," J. Phys. Soc. Jpn., vol. 40, pp. 921-924, Apr. 1970.

[7] E. Starikov, P. Shiktorov, V. Gruzinskis, L. Reggiani, L. Varani, J. C. Vaissiere, "Noise Enhancement as Indicator of Instability Onset in Semiconductor Structures," AIP. Conf. Proc., vol. 780, pp. 791-794, 2005.

[8] P. Shiktorov, E. Starikov, V. Gruzinskis, L. Varani, L. Reggiani, "Giant enhancement of low-frequency noise as precursor for the onset of a 
high-frequency instability," AIP Conf. Proc., vol. 1129, pp. 179-182, 2009.

[9] A. Íñiguez-de-la-Torre, I. Íñiguez-de-la-Torre, J. Mateos, T. González, "Correlation between low-frequency current-noise enhancement and high-frequency oscillations in GaN-based planar nanodiodes: A Monte Carlo study," Appl. Phys. Lett., vol. 99, p. 062109, 2011.

[10] Ó. García-Pérez, Y. Alimi. A. Song, I. Íñiguez-de-la Torre, S. Pérez, J. Mateos, T. González, "Experimental assessment of anomalous low- frequency noise increase at the onset of Gunn oscillations in InGaAs planar diodes," App. Phys. Lett., vol. 105, p. 113502, 2014.

[11] Agilent Technologies Inc., "High-accuracy noise figure measurements using the PNA-X series network analyzer," Application Note 1408-20 (5990-5800EN), 2013.

[12] D. M. Pozar, Microwave Engineering, NY: John Wiley and Sons, 1998, p. 551. 